\title{
Inhalt
}

Teil 1: Rechtliche Grundlagen der Unfallbegutachtung

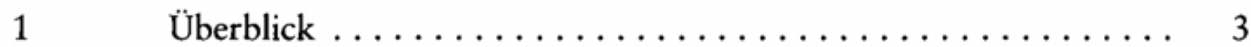

$2 \quad$ Gesetzliche Unfallversicherung $\ldots \ldots \ldots \ldots \ldots \ldots \ldots \ldots \ldots$

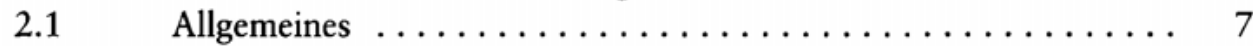

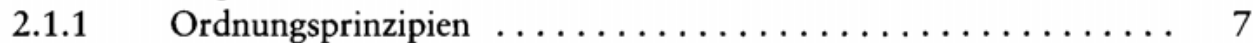

2.1.2 Berufsgenossenschaften und andere Unfallversicherungsträger $\ldots \quad 10$

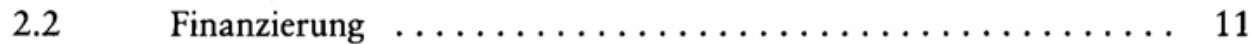

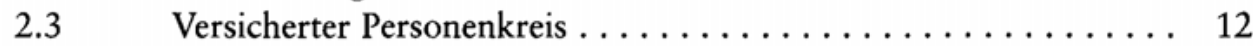

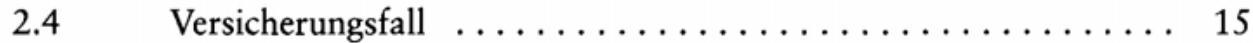

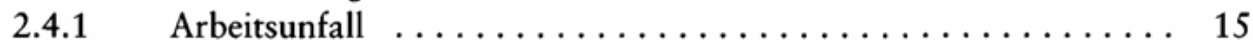

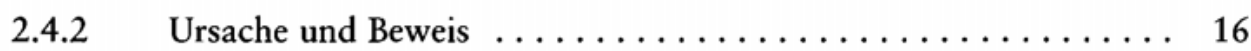

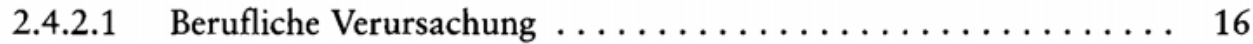

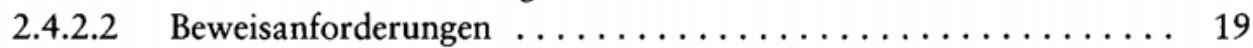

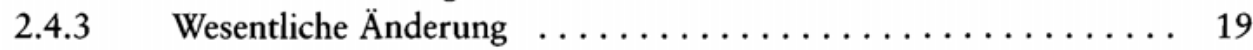

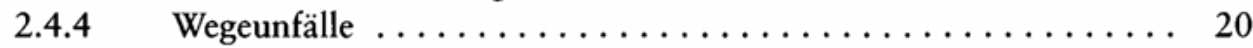

2.4.5 Berufskrankheiten ....................... 21

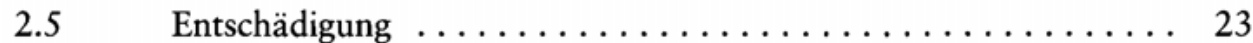

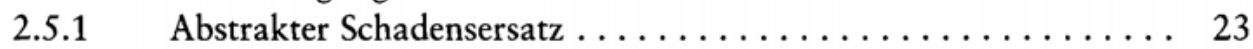

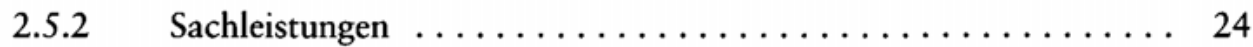

2.5.2.1 Heilbehandlung und medizinische Rehabilitation $\ldots \ldots \ldots \ldots 25$

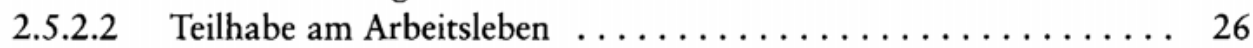

2.5.2.3 Teilhabe am Leben in der Gemeinschaft .............. 27

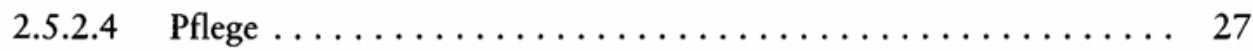

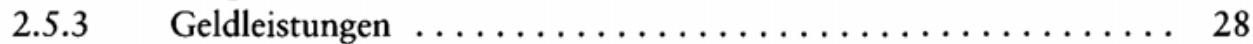

2.5.3.1 Verletztengeld - Übergangsgeld $\ldots \ldots \ldots \ldots \ldots \ldots \ldots \ldots \ldots 28$

2.5.3.2 Rente ............................ 29

2.5.3.3 Vorläufige Rente - „Dauerrente“ . . . . . . . . . . . 32

2.5.3.4 Rente bei Kindern in Kindergärten, Schülern und Studierenden . . 33

2.5.3.5 Leistungen an Hinterbliebene $\ldots \ldots \ldots \ldots \ldots \ldots \ldots \ldots . \ldots \ldots$

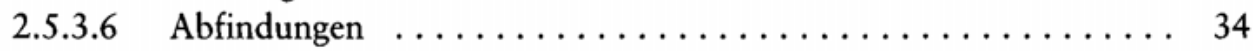

2.6 Verhältnis der Unfallversicherungsträger zu den Ärzten ...... 35

$2.7 \quad$ Verwaltungsverfahren $\ldots \ldots \ldots \ldots \ldots \ldots \ldots \ldots \ldots \ldots \ldots$

3 Gesetzliche Krankenversicherung $\ldots \ldots \ldots \ldots \ldots \ldots \ldots \ldots 4$

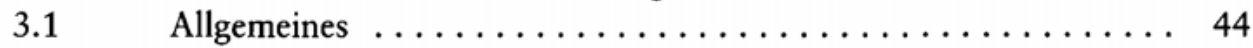

$3.2 \quad$ Träger der Krankenversicherung $\ldots \ldots \ldots \ldots \ldots \ldots \ldots \ldots 44$

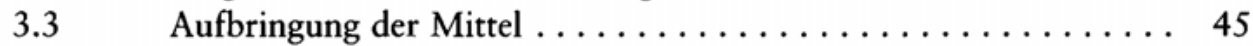




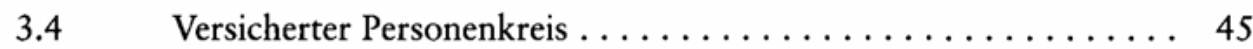

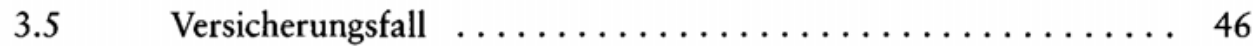

$3.6 \quad$ Leistungen der Krankenversicherung $\ldots \ldots \ldots \ldots \ldots \ldots \ldots 46$

$3.7 \quad$ Beziehungen zur Unfallversicherung $\ldots \ldots \ldots \ldots \ldots \ldots \ldots 47$

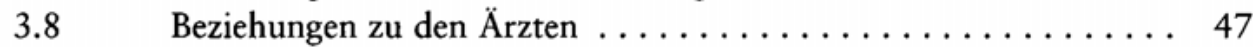

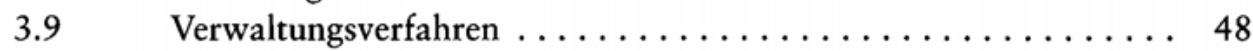

$4 \quad$ Gesetzliche Rentenversicherung $\ldots \ldots \ldots \ldots \ldots \ldots \ldots \ldots \ldots$

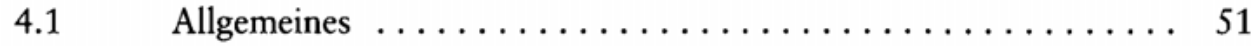

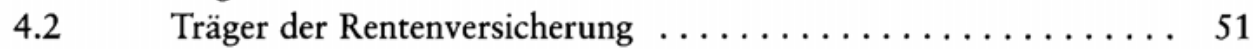

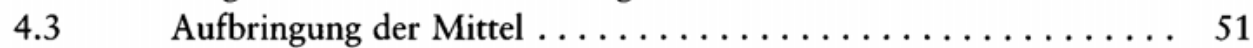

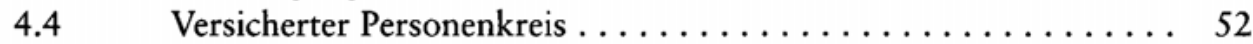

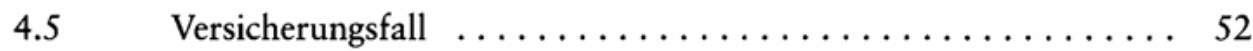

4.5.1 Minderung der Erwerbsfähigkeit $\ldots \ldots \ldots \ldots \ldots \ldots \ldots \ldots . \ldots \ldots$

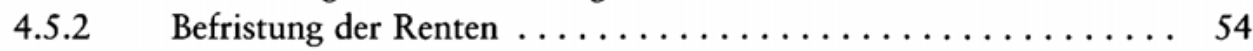

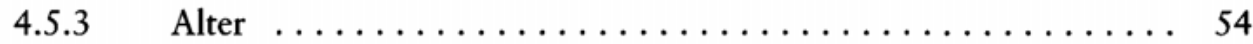

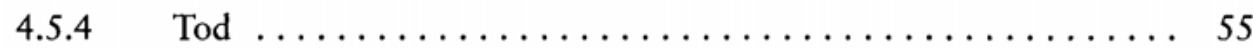

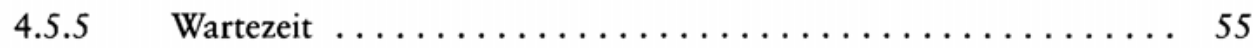

4.5.6 Besondere Versicherungsfälle der Knappschaftsversicherung .... 55

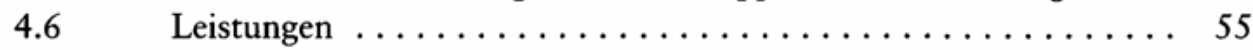

$4.7 \quad$ Beziehungen zur Unfallversicherung $\ldots \ldots \ldots \ldots \ldots \ldots \ldots$

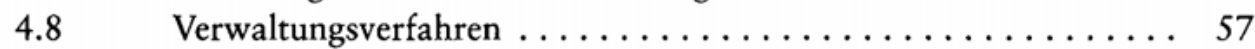

$5 \quad$ Gesetzliche Arbeitslosenversicherung $\ldots \ldots \ldots \ldots \ldots \ldots \ldots 58$

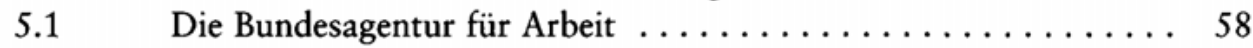

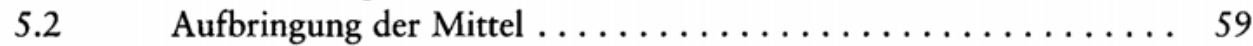

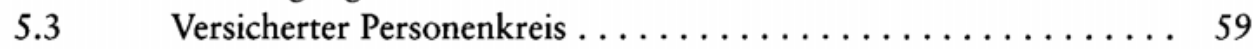

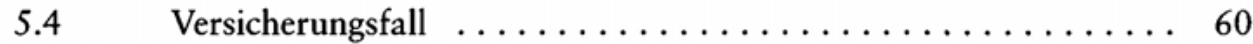

$5.5 \quad$ Leistungen zur Teilhabe am Arbeitsleben .............. 60

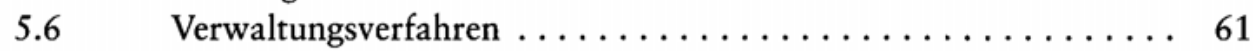

$5.7 \quad$ Beziehung zur gesetzlichen Unfallversicherung $\ldots \ldots \ldots \ldots \ldots 61$

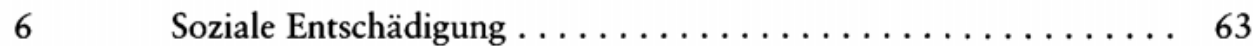

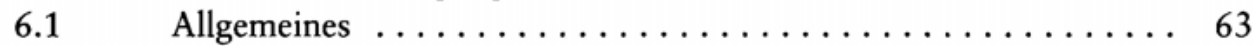

6.2 Soziale Entschädigung nach dem BVG ............. 63

6.3 Soziale Entschädigung nach anderen Gesetzen .......... 64

6.4 Umfang der Versorgungsleistungen bei Gesundheitsschäden . . . . 66

6.4.1 Heil- und Krankenbehandlung $\ldots \ldots \ldots \ldots \ldots \ldots \ldots \ldots 66$

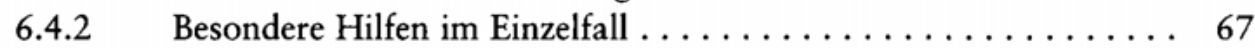

6.4.3 Renten und andere Geldleistungen $\ldots \ldots \ldots \ldots \ldots \ldots \ldots 68$

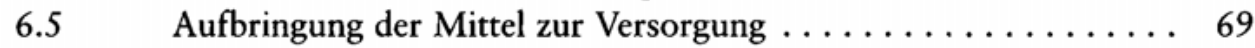

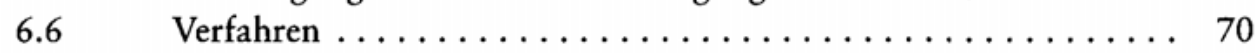

$6.7 \quad$ Hinweise für die Gutachter $\ldots \ldots \ldots \ldots \ldots \ldots \ldots \ldots \ldots$ 
$7 \quad$ Teilhabe von Menschen mit Behinderungen $\ldots \ldots \ldots \ldots \ldots \ldots 74$

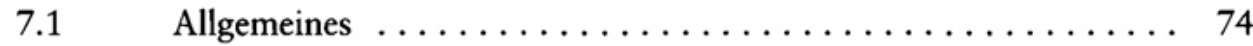

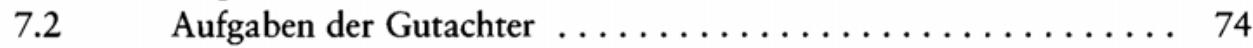

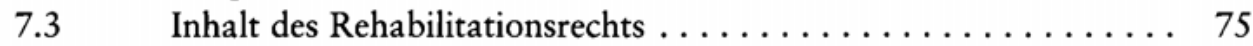

7.4 Beziehungen der Reha-Träger zu den Gutachtern ......... 76

$8 \quad$ Gesetzliche Pflegeversicherung $\ldots \ldots \ldots \ldots \ldots \ldots \ldots \ldots \ldots$

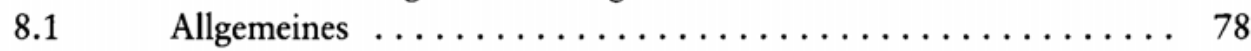

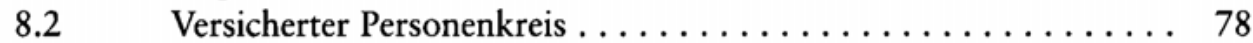

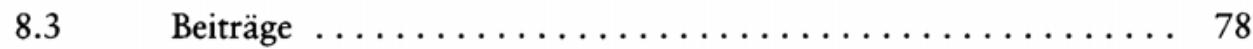

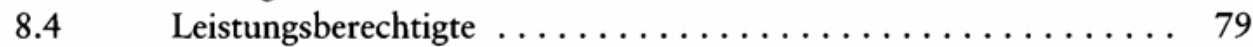

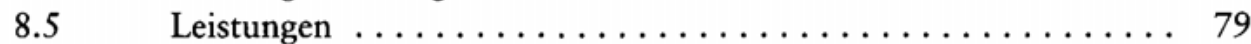

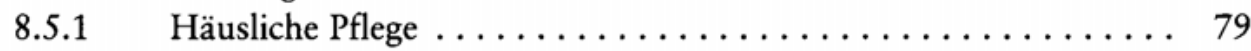

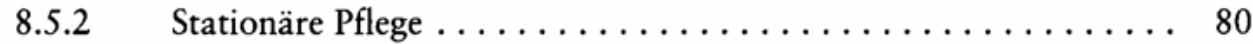

$8.6 \quad$ Begutachtung von Pflegebedürftigkeit $\ldots \ldots \ldots \ldots \ldots \ldots, 81$

$9 \quad$ Private Unfallversicherung $\ldots \ldots \ldots \ldots \ldots \ldots \ldots \ldots \ldots \ldots \ldots$

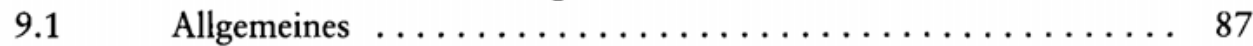

9.2 Träger der Versicherung - versicherter Personenkreis -

Aufbringung der Mittel $\ldots \ldots \ldots \ldots \ldots \ldots \ldots \ldots \ldots \ldots$

$9.3 \quad$ Versicherungsfall $\ldots \ldots \ldots \ldots \ldots \ldots \ldots \ldots \ldots \ldots \ldots \ldots$

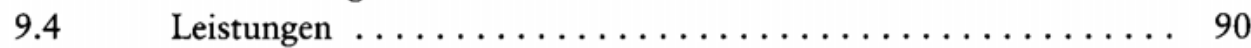

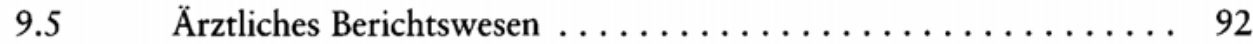

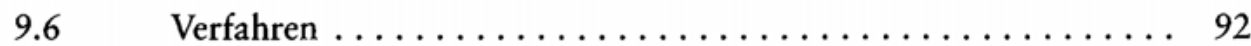

$10 \quad$ Vergütung von Gutachten $\ldots \ldots \ldots \ldots \ldots \ldots \ldots \ldots \ldots$

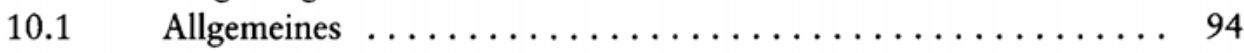

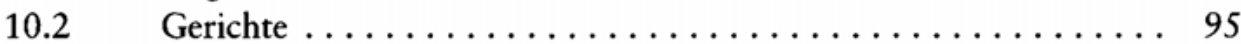

10.3 Gesetzliche Krankenversicherung . . . . . . . . . . 95

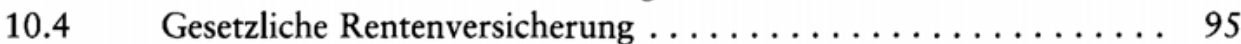

10.5 Bundesagentur für Arbeit $\ldots \ldots \ldots \ldots \ldots \ldots \ldots \ldots \ldots \ldots$

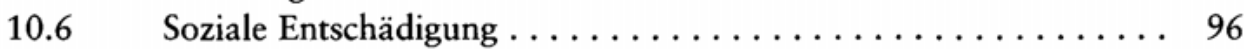

10.7 Gesetzliche Unfallversicherung $\ldots \ldots \ldots \ldots \ldots \ldots \ldots \ldots 96$

Teil 2: Medizinische Gesichtspunkte der Unfallbegutachtung

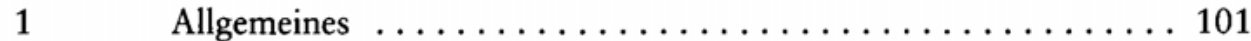

$1.1 \quad$ Gutachtertätigkeit der Ärzte $\ldots \ldots \ldots \ldots \ldots \ldots \ldots \ldots \ldots \ldots$

1.2 Rechtliche Stellung der Gutachter und ihre Aufgaben . . . . . . . 101

$1.3 \quad$ Formulierung der Gutachten $\ldots \ldots \ldots \ldots \ldots \ldots \ldots \ldots \ldots \ldots \ldots$

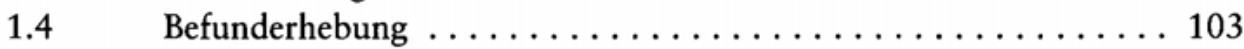

1.5 Entgegengesetzte Meinungen der Sachverständigen . . . . . . . . 104 
1.6 Untersuchung der Verletzten für die Begutachtung $\ldots \ldots \ldots \ldots 105$

1.7 Personenverwechslungen bei der Untersuchung $\ldots \ldots \ldots \ldots 105$

$1.8 \quad$ Fehler und Irrtümer im Gutachten $\ldots \ldots \ldots \ldots \ldots \ldots \ldots \ldots$

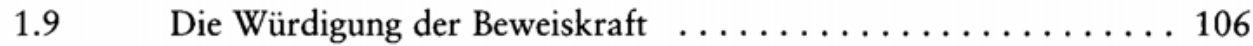

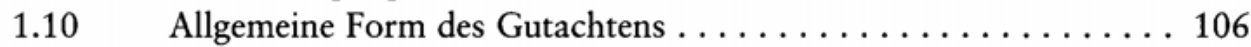

1.11 Ausstellung von Bescheinigungen und Zeugnissen . . . . . . 107

1.12 Auskunftspflicht der Ärzte . . . . . . . . . . . . . . . 107

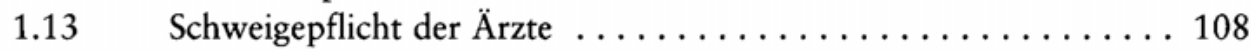

1.14 Sachkunde und Gutachternachwuchs . . . . . . . . . . . . 109

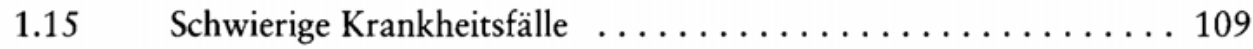

$1.16 \quad$ Vordruckgutachten ......................... 110

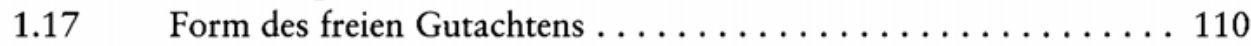

1.18 Die Minderung der Erwerbsfähigkeit (MdE) $\ldots \ldots \ldots \ldots \ldots 114$

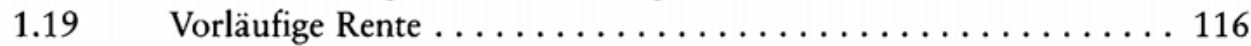

1.20 Dauerrente ............................. 116

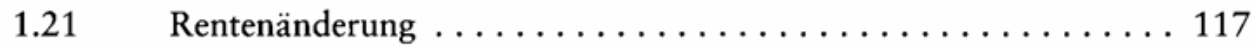

$1.22 \quad$ Vorschaden, Nachschaden . . . . . . . . . . . . . . 118

1.23 Wiederherstellende Behandlungsmaßnahmen -

1.24 Wichtige Untersuchungsmethoden für die Begutachtung . . . . . 120

1.25 Anleitung zur Benutzung der Messblätter und der Messung nach der Neutral-0-Methode . ................... 130

1.26 Simulation and Aggravation $\ldots \ldots \ldots \ldots \ldots \ldots \ldots \ldots \ldots \ldots$

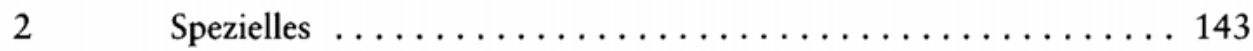

$2.1 \quad$ Die wichtigsten Rentensätze $\ldots \ldots \ldots \ldots \ldots \ldots \ldots \ldots \ldots \ldots \ldots \ldots$

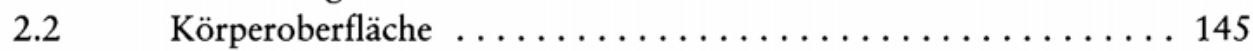

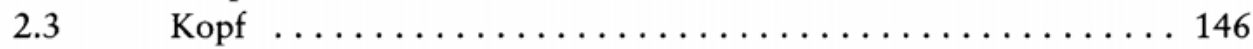

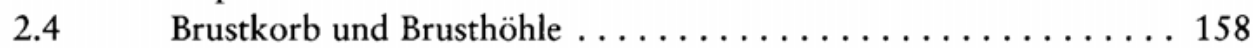

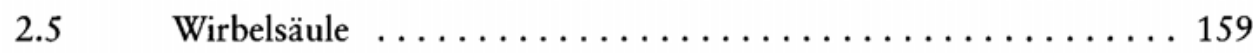

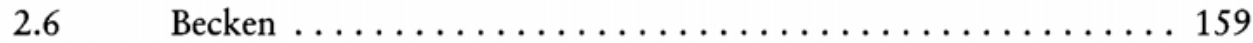

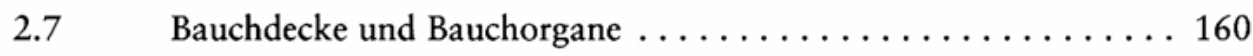

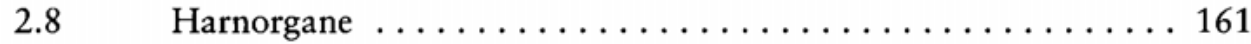

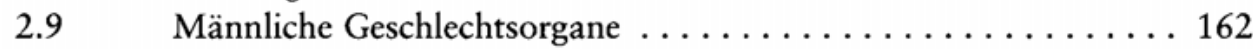

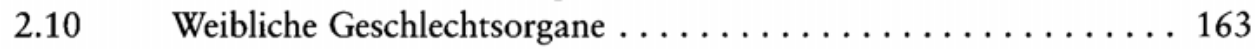

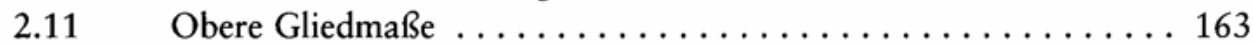

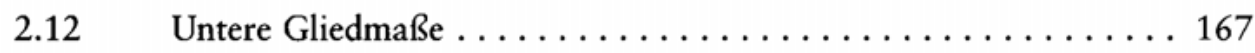

2.13 Anhaltspunkte zur Bemessung des Pflegegeldes gemäß $\$ 44$ Abs. 1 und Abs. 2 SGB VII ....................... 172

2.13.1 Zweck und inhaltliche Voraussetzungen des Pflegegeldes . . . . 172

2.13.1.1 Art und Schwere des Gesundheitsschadens . . . . . . . . . 173

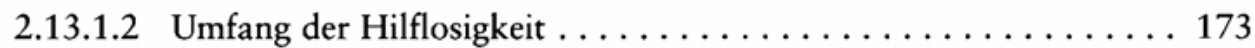

2.13.1.3 Einzelfallentscheidungen/Dokumentation und Erhebungsbogen . . 174 
2.14 Kategorien der Gesundheitsschäden und Einzeleinstufungen für die Festsetzung des Pflegegeldes bei Arbeitsunfällen und Berufskrankheiten

2.14.1 Kategorien der Gesundheitsschäden ................. 174

2.14.2 Einzeleinstufungen des Pflegegeldes bei Arbeitsunfälle (\$8 SGB VII)

3 Die Begutachtung von Fragen des ursächlichen Zusammenhanges zwischen Körperschäden und Arbeitsunfall ......... 195

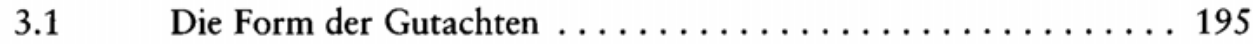

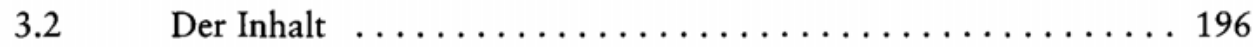

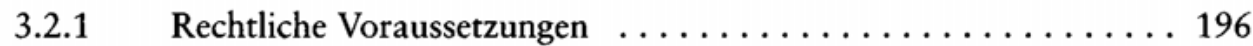

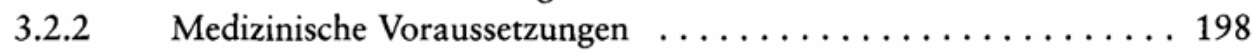

3.3 Spezielles über die Begutachtung von Zusammenhangsfragen . . . 199

3.3.1 Thermische Schädigungen . . . . . . . . . . . . . . . . . . . 199

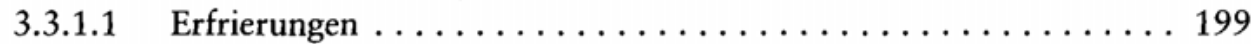

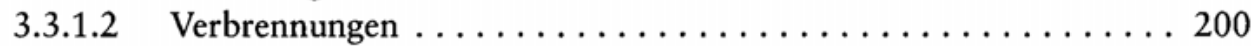

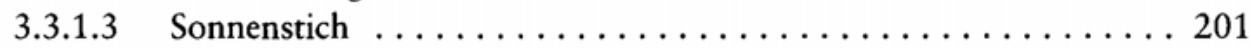

3.3.1.4 Hitzschlag . ......................... 201

3.3.2 Verletzungen durch Einwirkungen des elektrischen Stroms ..... 201

3.3.3 Akute Schädigungen durch Röntgenstrahlen, radioaktive Stoffe und andere ionisierende Strahlen ................. 201

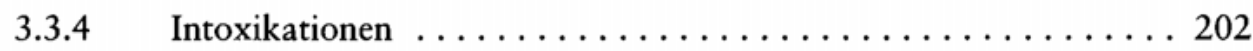

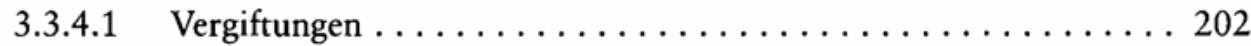

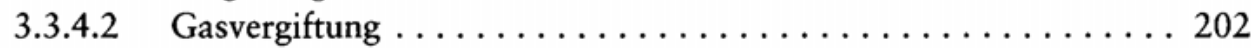

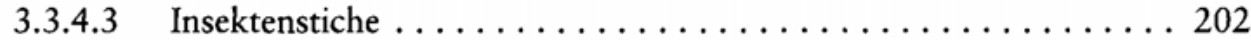

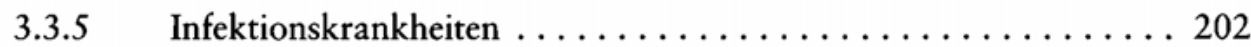

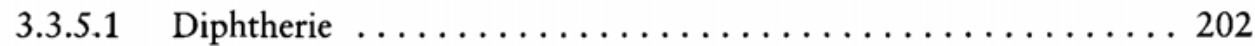

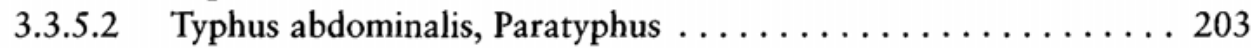

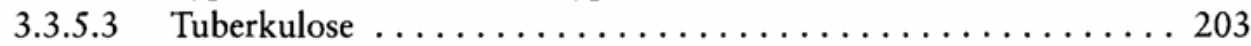

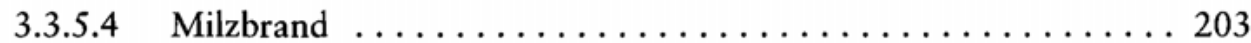

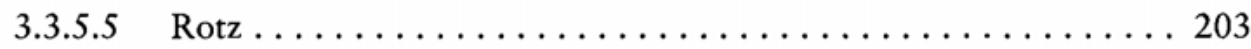

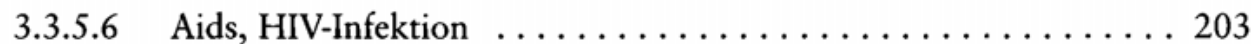

3.3.5.7 Hepatitis . . . . . . . . . . . . . . . . . . . 204

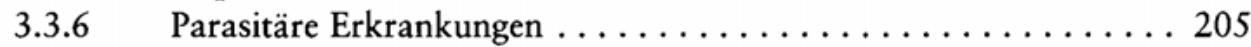

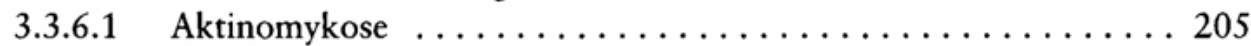

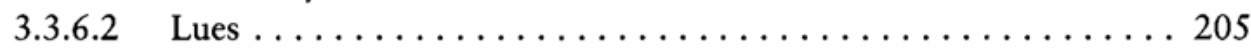

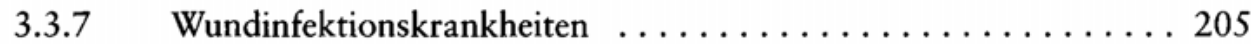

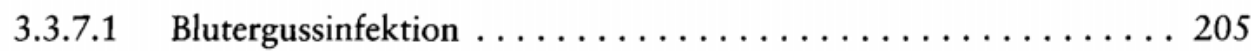

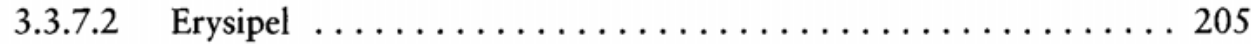

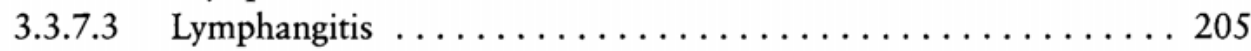

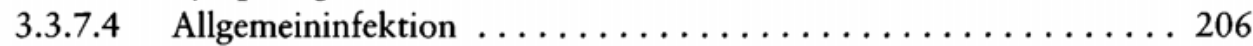

3.3.7.5 Tetanus .......................... 206 


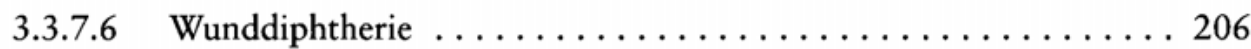

3.3.8 Geschwülste . . . . . . . . . . . . . . . . . 206

3.3.9 Stoffwechselkrankheiten und Krankheiten der endokrinen

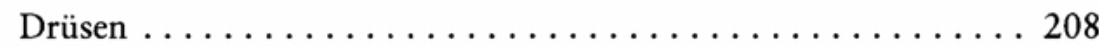

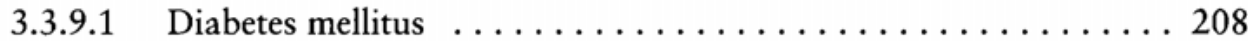

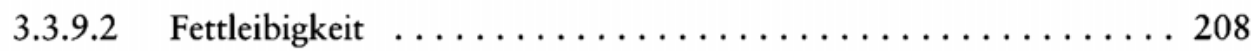

3.3.9.3 Nebennieren . . . . . . . . . . . . . . . . . . . . . . 209

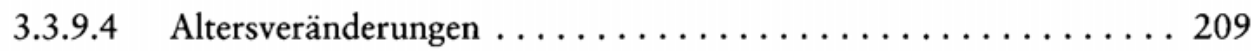

3.3.10 Erkrankungen des Blutes . . . . . . . . . . . . . . . . . . 209

3.3.10.1 Leukämie ............................... 209

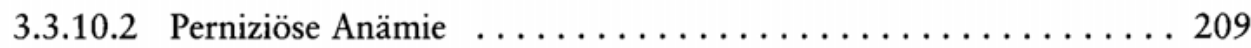

3.3 .10 .3 Milzzerreißungen $\ldots \ldots \ldots \ldots \ldots \ldots \ldots \ldots \ldots \ldots \ldots \ldots 210$

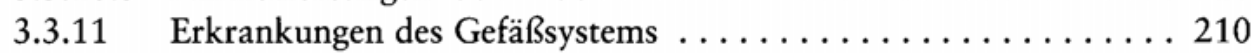

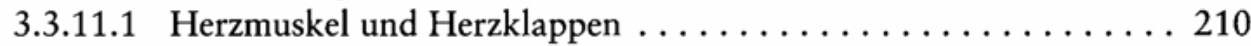

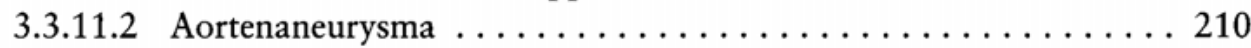

3.3.11.3 Aneurysmen peripherer Gefäße $\ldots \ldots \ldots \ldots \ldots \ldots \ldots \ldots \ldots 212$

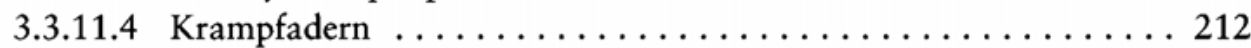

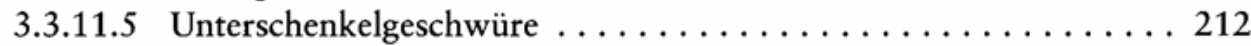

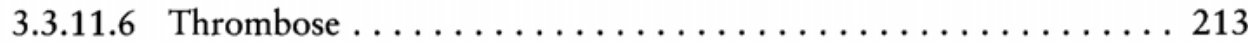

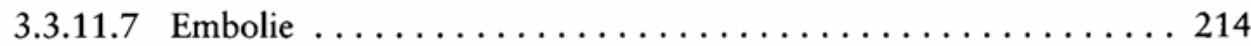

3.3.11.8 Arteriosklerose . . . . . . . . . . . . . . . . . . . . . 214

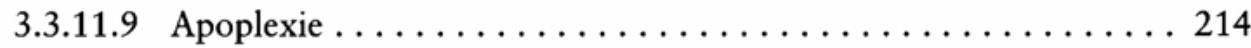

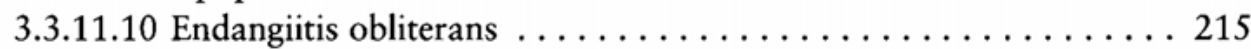

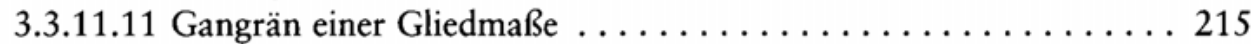

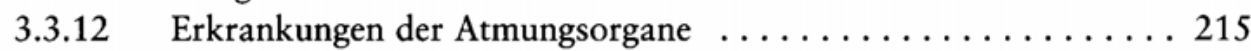

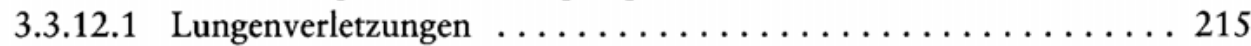

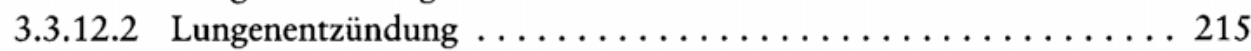

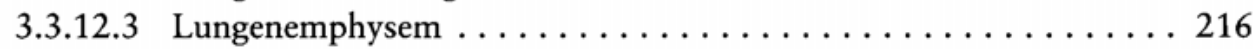

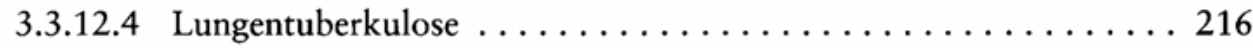

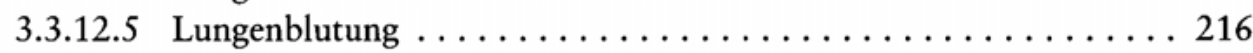

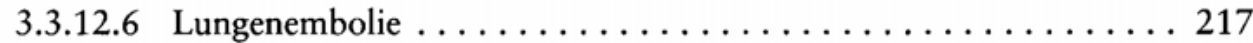

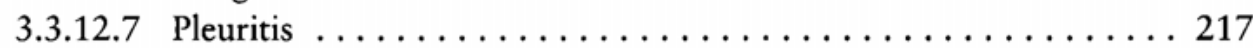

3.3.13 Erkrankungen der Bauchdecken $\ldots \ldots \ldots \ldots \ldots \ldots \ldots \ldots 217$

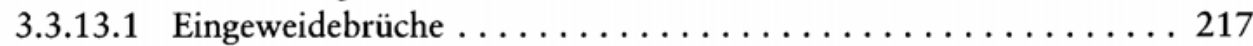

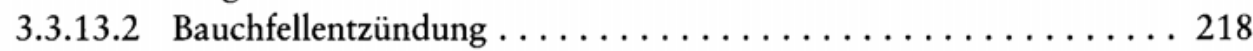

3.3.14 Erkrankungen des Magen- und Darmkanals . . . . . . . . 218

3.3.14.1 Ösophagusdivertikel $\ldots \ldots \ldots \ldots \ldots \ldots \ldots \ldots \ldots \ldots \ldots \ldots 218$

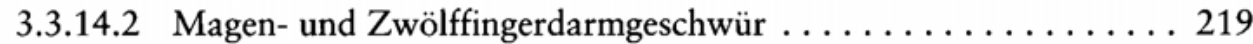

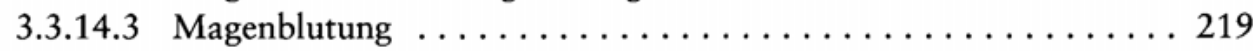

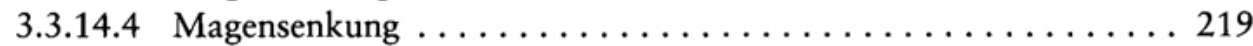

3.3 .14 .5 Magenkrebs ............................ 220

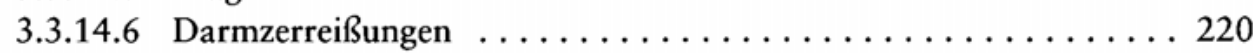

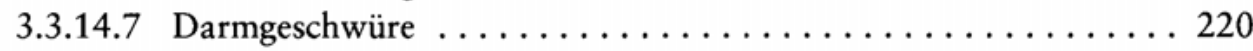




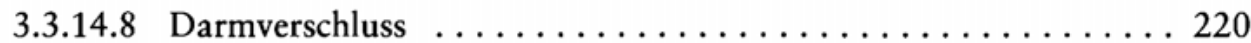

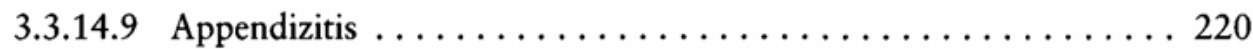

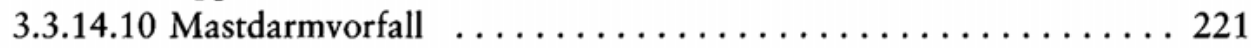

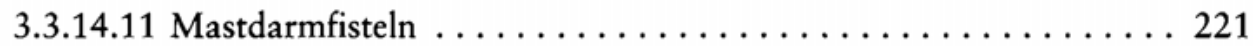

3.3.15 Erkrankungen der Leber, Gallenwege und Bauchspeicheldrüse . . 221

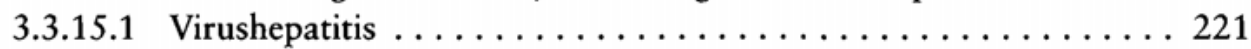

3.3.15.2 Chronische Hepatitis, Zirrhose $\ldots \ldots \ldots \ldots \ldots \ldots \ldots \ldots \ldots 221$

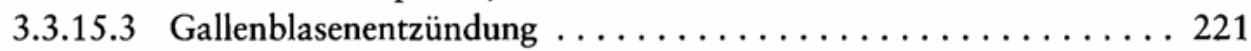

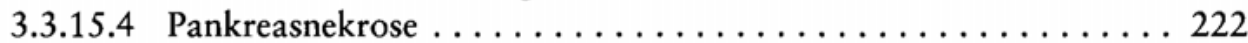

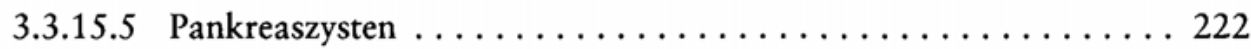

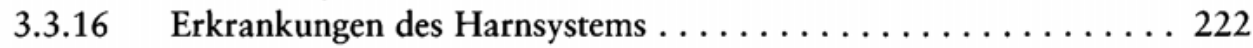

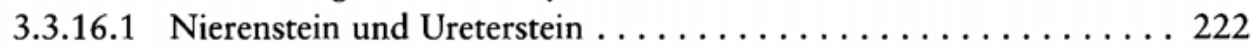

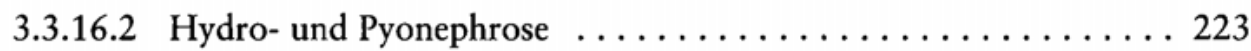

3.3.16.3 Neurogene Blasenentleerungsstörung $\ldots \ldots \ldots \ldots \ldots \ldots \ldots 223$

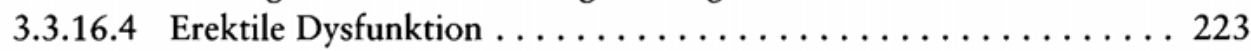

3.3.16.5 Nierentuberkulose . . . . . . . . . . . . . . . . . 224

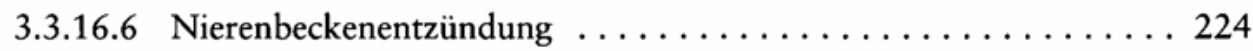

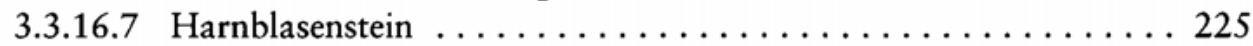

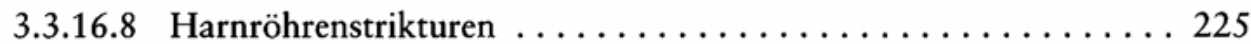

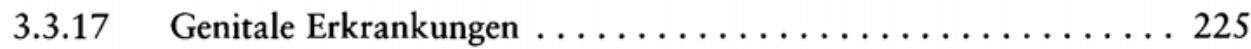

3.3.18 Erkrankungen der Haut und des Unterhautzellgewebes $\ldots \ldots \ldots 226$

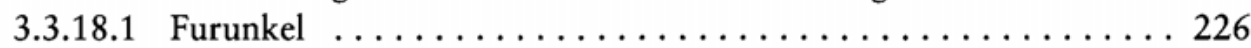

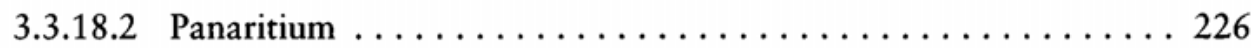

3.3.18.3 Zellgewebsentzündung (Phlegmon) $\ldots \ldots \ldots \ldots \ldots \ldots \ldots 226$

3.3.19 Erkrankungen der Muskeln, Sehnen und Schleimbeutel . . . . . 226

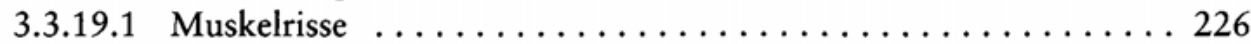

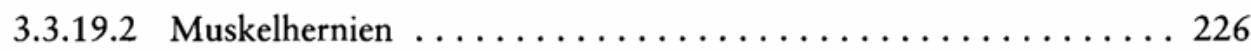

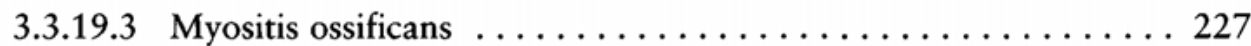

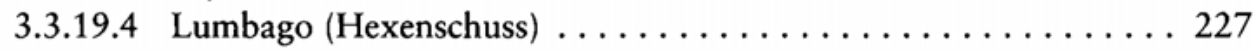

3.3.19.5 Bandscheibenvorfall im Bereich der Wirbelsäule . . . . . . . 227

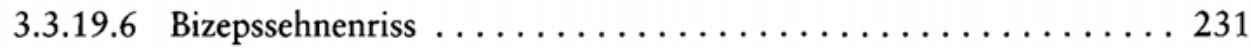

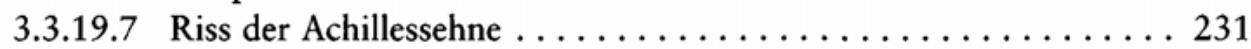

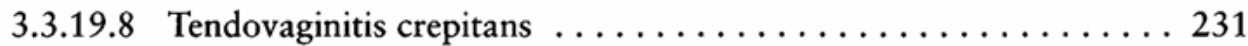

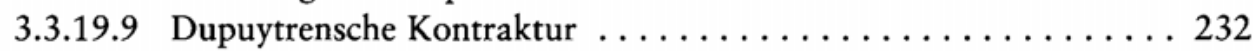

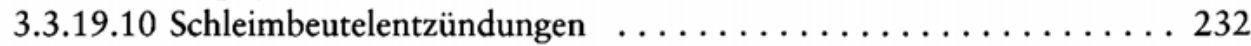

3.3.19.11 Periarthritis humero-scapularis $\ldots \ldots \ldots \ldots \ldots \ldots \ldots \ldots \ldots 232$

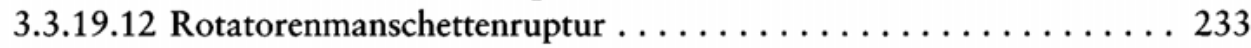

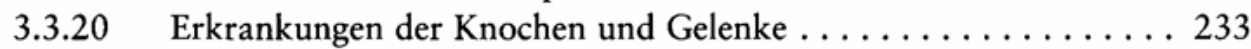

3.3.20.1 Akute Ostitis nach Weichteiltrauma $\ldots \ldots \ldots \ldots \ldots \ldots \ldots 233$

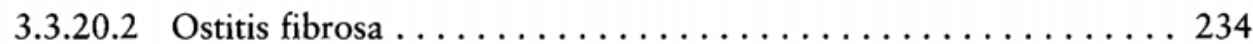

3.3.20.3 Tuberkulose der Knochen und Gelenke . . . . . . . . . . . 234

3.3.20.4 Spontanfrakturen ......................... 234

3.3.20.5 Dornfortsatzbruch (Schipperkrankheit) $\ldots \ldots \ldots \ldots \ldots \ldots 235$ 


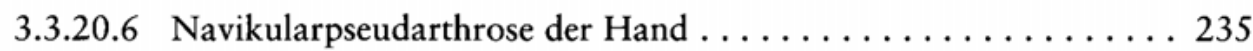

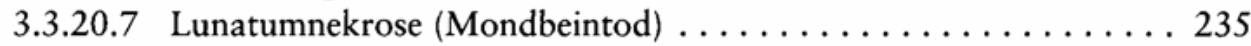

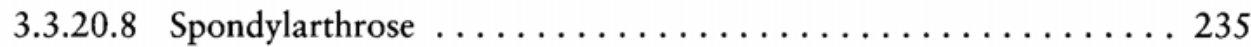

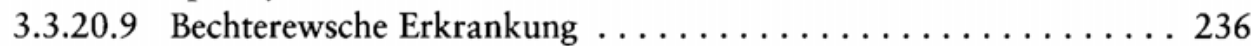

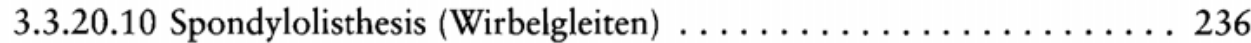

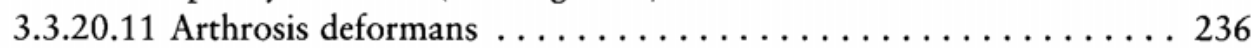

3.3.20.12 Traumatische Schultergelenksluxationen $\ldots \ldots \ldots \ldots \ldots \ldots 237$

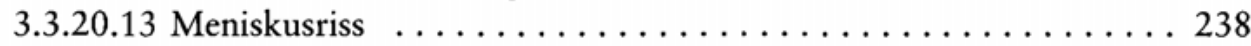

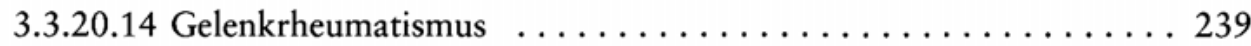

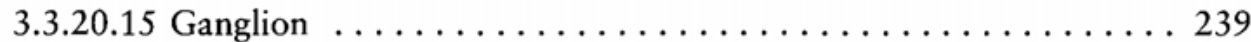

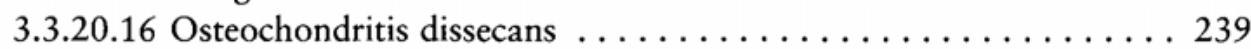

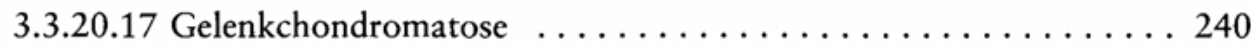

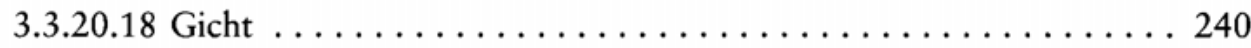

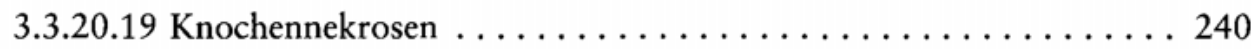

3.3.21 Erkrankungen des Nervensystems . . . . . . . . . . . . 241

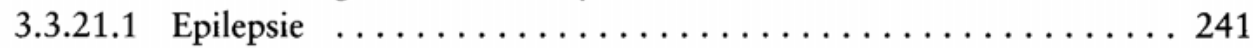

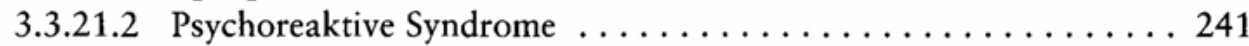

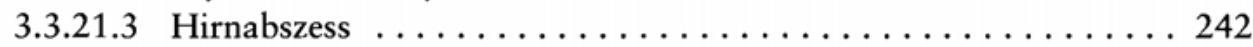

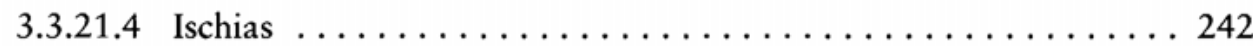

3.3.21.5 Traumatische Querschnittlähmung und Hämatomyelie . . . . . . 242

3.3.21.6 Neurofibromatose ....................... 244

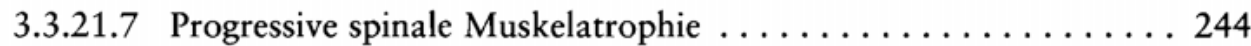

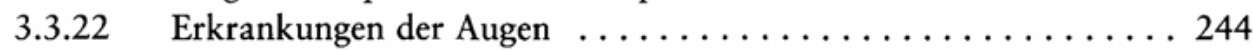

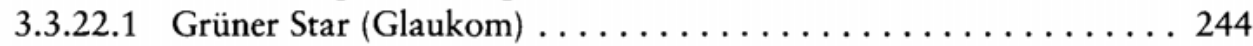

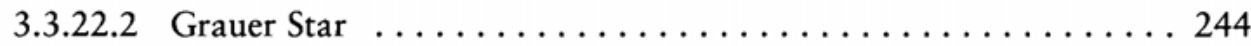

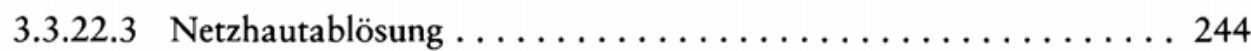

3.3.23 Berufsbedingte Erkrankungen der Wirbelsäule . . . . . . . . . 244

3.3.23.1 Überblick ............................. 244

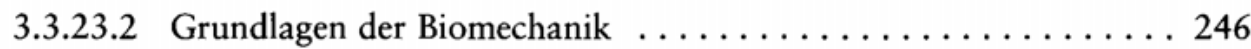

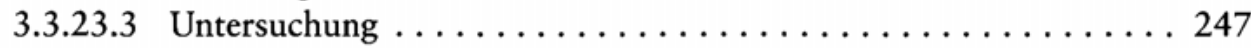

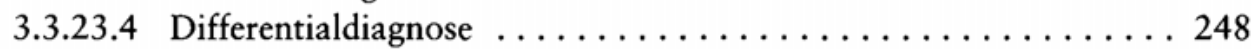

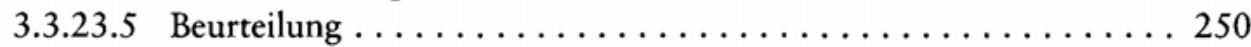

$4 \quad$ Psychische Störungen nach Unfällen $\ldots \ldots \ldots \ldots \ldots \ldots \ldots 251$

4.1 Besonderheiten der Begutachtung psychoreaktiver Verhaltens-

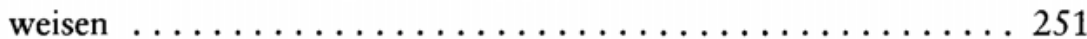

4.2 Begutachtungsmethodik bei psychischen Störungsbildern . . . . 252

4.3 Prüfkriterien der Unfallkausalität von psychischen Störungen . . . . 254

4.4 MdE-Maßstäbe für psychische Funktionsbeeinträchtigungen $\ldots 256$

$4.5 \quad$ MdE-Tabelle für psychische Gesundheitsschäden $\ldots \ldots \ldots \ldots 258$

$5 \quad$ Kriterien der medizinischen Beurteilung der Berufskrankheiten . . . 261

$5.1 \quad$ Liste der Berufskrankheiten $\ldots \ldots \ldots \ldots \ldots \ldots \ldots \ldots \ldots$ 
5.2 Merkblätter zu Berufskrankheiten (Auswahl) ........... 264

Nr. 1201 Erkrankungen durch Kohlenmonoxid ........... 264

Nr. 1202 Erkrankungen durch Schwefelwasserstoff ......... 266

Nr. 1303 Erkrankungen durch Benzol, seine Homologe und

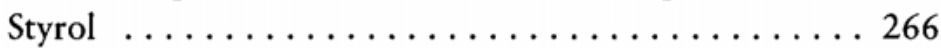

Nr. 1304 Erkrankungen durch Nitro- oder Aminoverbindungen des Benzols oder seiner Homologen oder ihrer Abkömmlinge ..................... 268

Nr. 1305 Erkrankungen durch Schwefelkohlenstoff . . . . . . . 269

Nr. 1306 Erkrankungen durch Methylalkohol (Methanol) . . . . 270

Nr. 1307 Erkrankungen durch organische Phosphor-

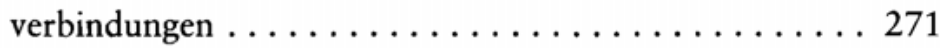

Nr. 1308 Erkrankungen durch Fluor oder seine Verbindungen ... 272

Nr. 1309 Erkrankungen durch Salpetersäureester . . . . . . . . 274

Nr. 1310 Erkrankungen durch halogenierte Alkyl-, Aryl- oder Alkylaryloxide ..................... 274

Nr. 1311 Erkrankungen durch halogenierte Alkyl-, Aryl- oder Alkylarylsulfide .................. 276

Nr. 1312 Erkrankungen der Zähne durch Säuren ......... 276

Nr. 1313 Hornhautschädigungen des Auges durch Benzochinon . . 277

Nr. 2101 Erkrankungen der Sehnenscheiden oder des Sehnengleitgewebes sowie der Sehnen- oder Muskelansätze, die zur Unterlassung aller Tätigkeiten gezwungen haben, die für die Entstehung, die Verschlimmerung oder das Wiederaufleben der Krankheit ursächlich waren oder sein können . . . . . 278

Nr. 2102 Meniskusschäden nach mehrjährigen andauernden oder häufig wiederkehrenden, die Kniegelenke überdurchschnittlich belastenden Tätigkeiten

Nr. 2103 Erkrankungen durch Erschütterung bei Arbeit mit Druckluftwerkzeugen oder gleichartig wirkenden Werkzeugen oder Maschinen ............... 279

Nr. 2104 Vibrationsbedingte Durchblutungsstörungen an den Händen, die zur Unterlassung aller Tätigkeiten gezwungen haben, die für die Entstehung, die Verschlimmerung oder das Wiederaufleben der Krankheit ursächlich waren oder sein können ....... 280

Nr. 2105 Chronische Erkrankungen der Schleimbeutel durch ständigen Druck $\ldots \ldots \ldots \ldots \ldots \ldots \ldots \ldots \ldots \ldots \ldots$

Nr. 2106 Druckschädigung der Nerven . . . . . . . . . 281 Nr. 2107 Abrissbrüche der Wirbelfortsätze . . . . . . . . . . 284 
Nr. 2108 Bandscheibenbedingte Erkrankungen der Lendenwirbelsäule durch langjähriges Heben oder Tragen schwerer Lasten oder durch langjährige Tätigkeit in extremer Rumpfbeugehaltung, die zur Unterlassung aller Tätigkeiten gezwungen haben, die für die Entstehung, die Verschlimmerung oder das Wiederaufleben der Krankheit ursächlich waren oder sein können . . . . . 285

Nr. 2109 Bandscheibenbedingte Erkrankungen der Halswirbelsäule durch langjähriges Tragen schwerer Lasten auf der Schulter, die zur Unterlassung aller Tätigkeiten gezwungen haben, die für die Entstehung, die Verschlimmerung oder das Wiederaufleben der Krankheit ursächlich waren oder sein können . . . . . . 289

Nr. 2110 Bandscheibenbedingte Erkrankungen der Lendenwirbelsäule durch langjährige, vorwiegend vertikale Einwirkung von Ganzkörperschwingungen im Sitzen, die zur Unterlassung aller Tätigkeiten gezwungen haben, die für die Entstehung, die Verschlimmerung oder das Wiederaufleben der Krankheit ursächlich waren oder sein können . 291

Nr. 2111 Erhöhte Zahnabrasionen durch mehrjährige quarzstaubbelastende Tätigkeit . . . . . . . . . . 295

Nr. 2201 Erkrankungen durch Arbeit in Druckluft . . . . . . . 296

Nr. 2301 Lärmschwerhörigkeit . . . . . . . . . . . . . 296

Nr. 2401 Grauer Star durch Wärmestrahlung . . . . . . . . . . 298

Nr. 2402 Erkrankungen durch ionisierende Strahlen . . . . . . . 299

Nr. 3101 Infektionskrankheiten, wenn der Versicherte im Gesundheitsdienst, in der Wohlfahrtspflege oder in einem Laboratorium tätig oder durch eine andere Tätigkeit der Infektionsgefahr in ähnlichem Maße

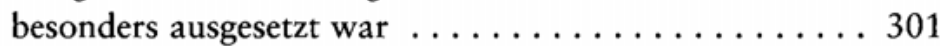

Nr. 3102 Von Tieren auf Menschen übertragbare Krankheiten . . . 305

Nr. 3104 Tropenkrankheiten, Fleckfieber . . . . . . . . . . . 307

Nr. 4302 Durch chemisch-irritativ oder toxisch wirkende Stoffe verursachte obstruktive Atemwegserkrankungen, die zur Unterlassung aller Tätigkeiten gezwungen haben, die für die Entstehung, die Verschlimmenrung oder das Wiederaufleben der Krankheit ursächlich waren

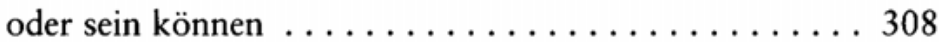

Nr. 5101 Schwere oder wiederholt rückfällige Hauterkrankungen, die zur Unterlassung aller Tätigkeiten gezwungen haben, die für die Entstehung, die Verschlimmerung oder das Wiederaufleben der Krankheit ursächlich waren oder sein können . . . . . . 309 
Anhang 1

Bildtafeln

\section{Anhang 2}

1 Die gesetzlichen Unfallversicherungsträger $\ldots \ldots \ldots \ldots \ldots \ldots \ldots \ldots$

2 Verzeichnis zur Ermittlung des zuständigen Unfallversicherungsträgers . . 348

3 Vertrag Ärzte/Unfallversicherungsträger (Ärztevertrag) . . . . . . . . . 354

4 Gemeinsame Richtlinien der Unfallversicherungsträger über Hilfsmittel

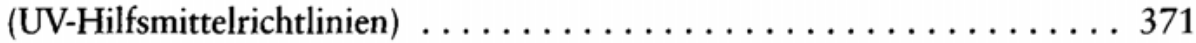

5 Gemeinsame Richtlinien der Spitzenverbände der Unfallversicherung über häusliche Krankenpflege ( $\$ 32$ Abs. 4 SGB VII) . . . . . . . . . . . . 379

6 Gemeinsame Richtlinien der Verbände der Unfallversicherungsträger

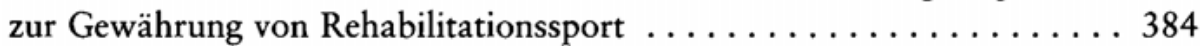

7 Gemeinsame Richtlinien der Bundesverbände der Unfallversicherungsträger über Belastungserprobung $\ldots \ldots \ldots \ldots \ldots \ldots \ldots \ldots \ldots$

8 Grundsätze des HVBG zur Förderung von Erholungsaufenthalten für Schwerstbehinderte der Unfallversicherungsträger . . . . . . . . . . . 391

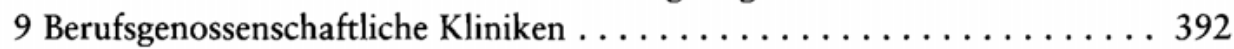

10 Publikationsverzeichnisse . . . . . . . . . . . . . . . . 394

Anhang 3

Allgemeine Versicherungsbedingungen für die Unfallversicherung -

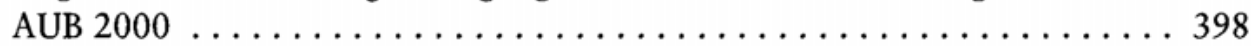

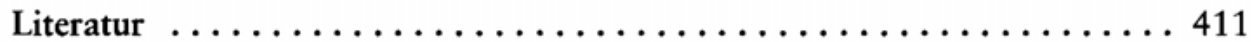

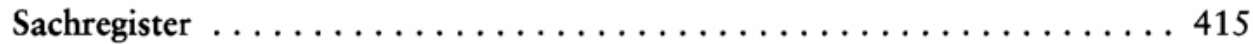


